\title{
Article \\ A Highly Divergent Hepacivirus Identified in Domestic Ducks Further Reveals the Genetic Diversity of Hepaciviruses
}

\author{
Xue-Lian Zhang ${ }^{\dagger}$, Xin-Yan Yao ${ }^{\dagger}$, Yu-Qian Zhang, Zhi-Hang Lv, Hong Liu, Jing Sun and Jian-Wei Shao *(D)
}

School of Life Science and Engineering, Foshan University, Foshan 528225, China; zxlsjw0312@163.com (X.-L.Z.); yaoxinyan95@163.com (X.-Y.Y.); aixxdxiaoqq@163.com (Y.-Q.Z.); lzh15637629901@163.com (Z.-H.L.); liuhong11252021@163.com (H.L.); sj1075963621@163.com (J.S.)

* Correspondence: jwshao1988@163.com

t These authors contributed equally to this work.

check for updates

Citation: Zhang, X.-L.; Yao, X.-Y.; Zhang, Y.-Q.; Lv, Z.-H.; Liu, H.; Sun, J.; Shao, J.-W. A Highly Divergent Hepacivirus Identified in Domestic Ducks Further Reveals the Genetic Diversity of Hepaciviruses. Viruses 2022, 14, 371. https://doi.org/ $10.3390 / v 14020371$

Academic Editor:

Helle Bielefeldt-Ohmann

Received: 26 January 2022

Accepted: 10 February 2022

Published: 11 February 2022

Publisher's Note: MDPI stays neutral with regard to jurisdictional claims in published maps and institutional affiliations.

Copyright: (C) 2022 by the authors. Licensee MDPI, Basel, Switzerland. This article is an open access article distributed under the terms and conditions of the Creative Commons Attribution (CC BY) license (https:// creativecommons.org/licenses/by/ $4.0 /)$.

\begin{abstract}
Hepaciviruses represent a group of viruses that pose a significant threat to the health of humans and animals. During the last decade, new members of the genus Hepacivirus have been identified in various host species worldwide, indicating the widespread distribution of genetically diversified hepaciviruses among animals. By applying unbiased high-throughput sequencing, a novel hepacivirus, provisionally designated Hepacivirus $Q$, was discovered in duck liver samples collected in Guangdong province of China. Genetic analysis revealed that the complete polyprotein of Hepacivirus $Q$ shares $23.9-46.6 \%$ amino acid identity with other representatives of the genus Hepacivirus. Considering the species demarcation criteria for hepaciviruses, Hepacivirus $Q$ should be regarded as a novel hepacivirus species of the genus Hepacivirus within the family Flaviviridae. Phylogenetic analyses also indicate the large genetic distance between Hepacivirus $Q$ and other known hepaciviruses. Molecular detection of this novel hepacivirus showed an overall prevalence of $15.9 \%$ in duck populations in partial areas of Guangdong province. These results expand knowledge about the genetic diversity and evolution of hepaciviruses and indicate that genetically divergent hepaciviruses are circulating in duck populations in China.
\end{abstract}

Keywords: novel hepacivirus; genetic diversity; domestic duck; China

\section{Introduction}

The genus Hepacivirus, along with the genera Flavivirus, Pegivirus, and Pestivirus, currently belongs to the family Flaviviridae, which comprises a genetically diverse group of human and animal pathogens [1]. Members of the genus Hepacivirus are enveloped viruses, with unsegmented, single-stranded, positive-sense RNA genomes about $10 \mathrm{~kb}$ in length. The genomes of these viruses contain a single long open reading frame (ORF) encoding a single polyprotein of about 3000 amino acids (aa), which is flanked by a $5^{\prime}$ untranslated region (UTR) and a $3^{\prime}$ UTR [1]. This polyprotein is further cleaved by host and viral proteases into structural proteins (Core, E1, and E2) and nonstructural proteins (p7, NS2, NS3, NS4A, NS4B, NS5A, and NS5B) [2,3].

Hepatitis $C$ virus (HCV), the type species of the genus Hepacivirus, is one of the leading causes of acute and chronic hepatitis, liver cirrhosis, and hepatocellular carcinoma in humans, and the infection rate of HCV worldwide is approximately $3 \%$, with an estimated 58 million people suffering from chronic HCV infection (https: / /www.who.int/en/newsroom/fact-sheets / detail/hepatitis-c; accessed on 27 July 2021). Since its first discovery in 1989, HCV has been considered as the sole representative of the genus Hepacivirus, and humans were regarded as the only natural host for a long time, although experimental infection of HCV in chimpanzees is possible [4]. However, since 2011, many novel genetically diversified hepaciviruses have been discovered from a wide range of mammalian hosts, such as dogs [5], horses [5], monkeys [6], bats [7], rodents [8,9], cattle [10,11], and donkeys [12]. In addition, non-mammalian hosts harboring hepaciviruses have also been 
described, including catshark [13], birds [14,15], fish, and other vertebrates [16-18]. Moreover, some highly divergent hepaciviruses were recently detected in mosquitoes and ticks, although their true host is uncertain [19-21]. Furthermore, a novel hepacivirus tentatively named RHV-GS2015 was recently identified in long-tailed ground squirrels in China [22]. Currently, these divergent hepaciviruses identified in different host species have been classified into 14 formal species (named Hepacivirus $A-N$ ) and some unclassified hepaciviruses, based on their phylogenetic relationships and host range [1].

Hepaciviruses in birds were first identified in domestic ducks collected over a wide geographical area of China in 2019, with the positive rate of viral RNA in the different regions ranging from 38.5-88\% [14]. This infection may be associated with severe drops in egg production [14]. Since then, hepaciviruses have also been detected in the Guangdong province of China [23]. Furthermore, novel hepaciviruses were detected in Bald eagles in the USA in 2019 [15]. In the present study, a novel highly divergent hepacivirus, provisionally designated Hepacivirus $Q$, was identified in liver samples of domestic ducks collected in Guangdong province, China through an unbiased high-throughput sequencing and metatranscriptomic analysis. We obtained the complete genome sequence and determined the genomic features, the genetic diversity, and the evolution of Hepacivirus $Q$. In addition, the prevalence of this novel hepacivirus in duck populations in partial areas of Guangdong province in China was also investigated. This study expands knowledge about the genetic diversity and evolution of hepaciviruses and shows that genetically divergent hepaciviruses are circulating in duck populations in China.

\section{Materials and Methods}

\subsection{Sample Collection, RNA Extraction, and Meta-Transcriptome Sequencing}

In April 2021, 30 liver tissues of domestic ducks were collected by authorized veterinarians of the veterinary hospital of Foshan University from a duck farm located in Zhaoqing city of Guangdong province. Approximately $50 \mathrm{mg}$ of liver tissue was homogenized with $500 \mu \mathrm{L}$ sterile phosphate-buffered saline (PBS), and total RNA was extracted from $200 \mu \mathrm{L}$ homogenates using TRIzol LS reagent (Invitrogen, Carlsbad, CA, USA) and subsequently purified using the RNeasy Plus Mini Kit (Qiagen, Hilden, Germany). The quantity and quality of extracted RNA was evaluated with a NanoDrop 2000 (Thermo Fisher Scientific, Waltham, MA, USA). Subsequently, all RNA solutions for individual homogenates were then merged as one pool in equal quantity, and the quality of the pooled RNA was evaluated using an Agilent 2100 Bioanalyzer (Agilent Technologies, Santa Clara, CA, USA) before library construction and sequencing.

RNA library preparation was conducted following the methodology previously described [16,24]. Briefly, ribosomal RNA (rRNA) was depleted using a Ribo-Zero-Gold (Epidemiology) kit (Illumina Inc., San Diego, CA, USA), following the manufacturer's instructions. The rest of the RNA was fragmented, reverse-transcribed, adapted, and purified using a TruSeq total RNA library preparation kit (Illumina Inc.). Library quality was examined by the Qubit (Thermo Fisher Scientific) high-sensitivity RNA/DNA assays and Agilent 2100 Bioanalyzer (Agilent Technologies). Paired-end (150 bp) sequencing was performed on the Illumina Hiseq 2500 platform. All library preparation and sequencing were performed by Novogene (Tianjin, China).

\subsection{Bioinformatics Analyses and Genome Sequence Determination}

Sequencing reads were demultiplexed, trimmed for the adaptor and quality control with the fastp program [25], and subsequently de novo assembled using the Megahit program [26] with default parameter settings. The resulting contigs were compared against the entire non-redundant protein (nr) database downloaded from GenBank using the diamond blastx program [27] with an e-value threshold of $1 \times 10^{-4}$. These viral contigs with unassembled overlaps or from the same scaffold were merged using the SeqMan program implemented in the Lasergene software package (version 7.1, DNAstar). To confirm the assembly results, reads were mapped back to the target contigs with Bowtie2 [28], and 
assembly errors were inspected using the Integrated Genomics Viewer (IGV) [29]. Gaps between these contigs were filled by RT-PCR and Sanger sequencing. The genome termini of the virus were determined using $5^{\prime} / 3^{\prime}$ RACE kits (TaKaRa, Dalian, China), as described previously [30]. The final virus genome sequences were obtained for the majority consensus of the mapping assembly and confirmed by Sanger sequencing with overlapping primers that covered the entire sequence.

\subsection{PCR Screening for This Newly Identified Hepacivirus}

A total of 240 liver samples were collected from domestic ducks without obvious abnormalities in Zhaoqing $(n=38)$, Qingyuan $(n=50)$, Foshan $(n=66)$, and Jiangmen $(n=86)$ cities of Guangdong province. Moreover, the 30 individual samples collected in Zhaoqing that were subjected to meta-transcriptome sequencing were also included in the investigation into the prevalence of this novel hepacivirus described herein in duck populations (Figure 1). Total RNA was prepared from $200 \mu \mathrm{L}$ of homogenates of individual liver samples and subjected to the screening of Hepacivirus $Q$ by nested PCR using the specific primer pairs targeting the NS5B coding region (Table S1). All the PCR products of the expected size were confirmed by Sanger sequencing.

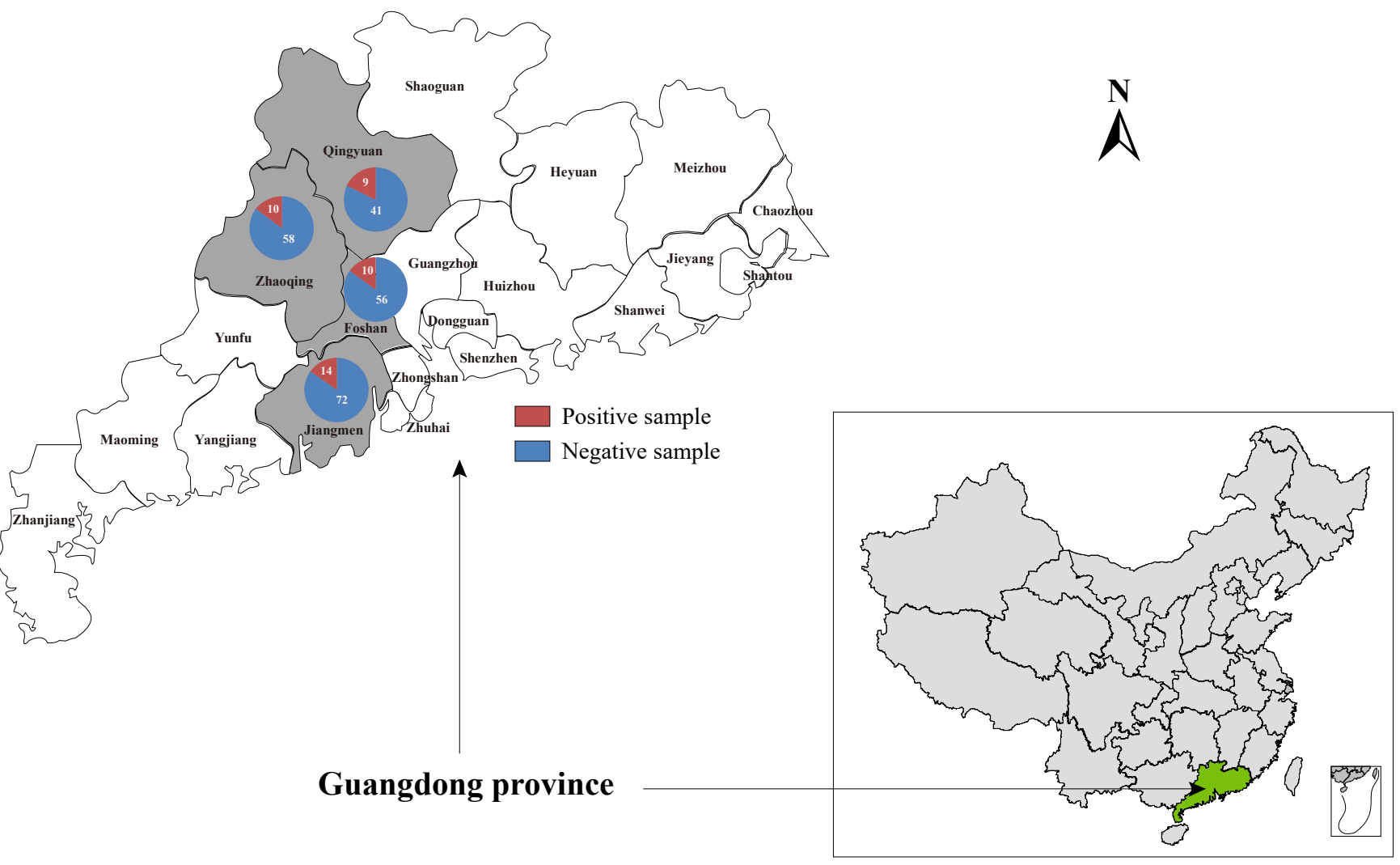

Figure 1. Geographic maps showing the location of sampling sites where the duck liver samples in this study were collected. This map was plotted with a combination of Surfer software version 4 (Golden Software, Golden, CO, USA) and Adobe illustrator version CC2017 (Adobe, San Jose, CA, USA).

\subsection{Sequence Comparison and Phylogenetic Analyses}

The prediction of potential open reading frames (ORFs) was performed by ORFfinder (https://ftp.ncbi.nlm.nih.gov/genomes/TOOLS/ORFfinder/linux-i64/; accessed on 4 May 2021) and annotated based on comparisons against the non-redundant protein database. The cleavage sites for the viral polyprotein processing were extrapolated by manually comparing the polyprotein sequence with that with the highest amino acid sequence identity, which was previously described in the Bald eagle hepacivirus (BeHV) identi- 
fied in the USA [15]. N-glycosylation sites were predicted using NetNGlyc 1.0 (http: //www.cbs.dtu.dk/services/NetNGlyc; accessed on 18 March 2012). The sequences of viruses classified to the genus Hepacivirus were downloaded from GenBank, and amino acid (aa) sequence identities between the sequences generated in this study along with other reference sequences were calculated by the MegAlign program available in the Lasergene software package (version 7.1, DNAstar, Madison, WI, USA).

To infer the phylogenetic relationship between the viruses identified in the present study and other known hepaciviruses, amino acid sequences of the complete polyprotein, NS3 (peptidase and helicase), and NS5 (RNA-dependent RNA polymerase) proteins were aligned using the E-INS-i algorithm implemented in the MAFFT program [31]. Phylogenetic trees were then reconstructed using the maximum likelihood method (ML) implemented in PhyML version 3.0 [32], employing the LG amino acid substitution model with a gamma (Г)-distribution model (i.e., LG $+\Gamma$ ), and determined with Prot-Test 3 [33] and a Subtree Pruning and Regrafting (SPR) branch-swapping algorithm with bootstrap support values calculated from 100 replicate trees. All phylogenetic trees were mid-point rooted for purposes of clarity only.

\subsection{Genome Recombination Analysis}

The RDP, GENECONV, bootscan, maximum chi square, Chimera, SISCAN, and distance plot recombination detection methods available within RDP4 [34] were used to determine the potential recombination events that occurred in the evolutionary history of this newly identified hepacivirus. The analyses were performed based on the complete genome sequences with default settings for the different test methods and a Bonferroni corrected $p$-value cutoff of 0.05 . Only sequences with significant evidence $(p<0.05)$ of recombination detected by at least two methods and confirmed by phylogenetic analysis were taken to represent strong evidence for recombination. Additionally, similarity plot analyses were inferred to further characterize potential recombination events, including the location of possible breakpoints, as implemented in Simplot version 3.5.1 [35].

\section{Results}

\subsection{Identification of a Novel Hepacivirus in Domestic Ducks}

Pooled RNA samples extracted from 30 individual duck liver samples were screened for known and putative novel viruses by unbiased high-throughput RNA sequencing. Through de novo assembly and comparison against the $\mathrm{nr}$ database using the diamond blastx program with an e-value cutoff of $1 \times 10^{-4}$, six contigs ranging from 455 to 3016 $\mathrm{nt}$ in length were annotated as the Bald eagle hepacivirus of the genus Hepacivirus within the family Flaviviridae, with $37.4-65.1 \%$ amino acid identity. After filling the gaps between the contigs through RT-PCR and determining the terminal sequences using $5^{\prime} / 3^{\prime}$ RACE, this virus's complete genome sequence was obtained. Using this sequence as the reference sequence, 563 reads were remapped to this reference sequence and provided $99.0 \%$ genome coverage (9798 nt/9893 nt) with $96.8 \%$ pairwise identity at a mean depth of $8.5 \times$ (Figure 2).

The complete genome sequence contained one ORF of 9000 nucleotides which encodes a polyprotein of 2999 amino acids (aa). The blastp search against the NCBI nr database revealed highly significant homology to the polyprotein of a hepacivirus named Bald eagle hepacivirus (BeHV). Alignments were contiguous and extended over the entire length of the polyprotein (query coverage, 100\%). Results from pairwise alignments between the proteins of this newly identified virus and other known hepacivirus species are summarized in Table 1. The complete polyprotein of this novel hepacivirus shared $23.9 \%-46.6 \%$ amino acid identity with other representatives of the genus Hepacivirus. The most highly conserved regions were those encoding the NS3 and NS5B proteins, with $35.6-53.0 \%$ and $30.9-56.3 \%$ amino acid identity, respectively (Table 1 ). In the conserved region of NS3 (positions 1123-1566) and NS5B (amino acid positions 2536-2959), as numbered in the reference sequence of Hepacivirus $C$ (NC038882), this novel virus identified herein exhibited amino acid $p$-distances of $0.463-0.600$ and $0.395-0.654$, respectively, with the known 
hepaciviruses (Table S2), which exceed the species demarcation cutoff for hepacivirus [1]. The overall pattern and degree of sequence diversity suggest that the new virus may be considered a novel species of the genus Hepacivirus. Following the nomenclature used for previous species, this novel virus determined in the present study was provisionally named "Hepacivirus Q".

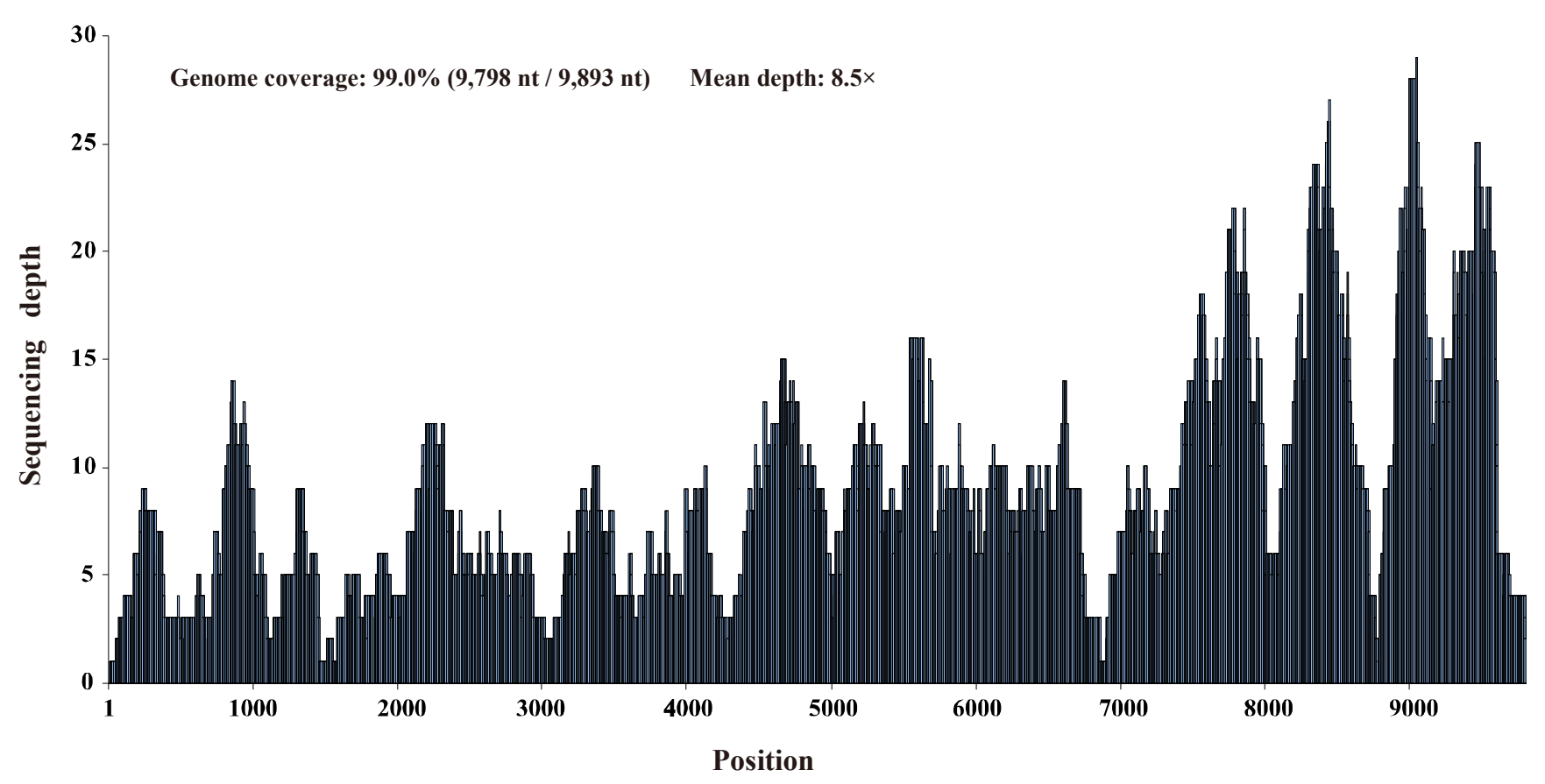

Figure 2. Mapped read count plot of the Hepacivirus $Q$ genome. The histograms show the coverage depth per base of the Hepacivirus $Q$ genome, and the mean sequencing depth was $8.5 \times$.

Table 1. Amino acid sequence similarity of mature Hepacivirus $Q$ proteins compared to those of other hepaciviruses. Sequences were aligned using the ClustalW method and the calculated sequence similarity implemented in MegAlign.

\begin{tabular}{|c|c|c|c|c|c|c|c|c|c|c|c|c|c|c|c|c|c|c|c|}
\hline \multirow[b]{2}{*}{ Protein } & \multicolumn{19}{|c|}{ Value for the Indicated Virus in Relation to Hepacivirus $Q$} \\
\hline & A & B & $\mathrm{C}$ & D & $\mathbf{E}$ & $\mathbf{F}$ & G & $\mathbf{H}$ & $\mathbf{I}$ & $\mathbf{J}$ & K & $\mathbf{L}$ & $\mathbf{M}$ & $\mathbf{N}$ & RHV & $\mathrm{JgV}$ & HCL1 & GD61 & BeHV \\
\hline Polyprotein & 23.9 & 24.6 & 23.7 & 24.6 & 24.8 & 24.0 & 24.0 & 24.0 & 24.1 & 24.7 & 24.6 & 25.5 & 25.4 & 24.1 & 25.0 & 39.1 & 35.5 & 35.6 & 46.6 \\
\hline Core & 26.5 & 24.1 & 23.9 & 25.2 & 24.7 & 28.1 & 26.5 & 26.5 & 23.9 & 28.6 & 25.8 & 24.8 & 27.5 & 23.1 & 24.1 & 9.4 & 28.8 & 28.2 & 47.6 \\
\hline E1 & 24.6 & 20.8 & 19.4 & 19.7 & 22.5 & 19.3 & 18.5 & 17.6 & 18.7 & 26.8 & 22.9 & 16.0 & 23.9 & 16.1 & 18.2 & 45.2 & 38.6 & 38.6 & 55.6 \\
\hline E2 & 11.6 & 13.2 & 15.0 & 15.0 & 15.9 & 16.5 & 17.0 & 15.2 & 12.8 & 9.3 & 12.1 & 15.0 & 12.5 & 13.7 & 14.8 & 29.7 & 30.9 & 31.7 & 44.6 \\
\hline p7 & 21.0 & 17.5 & 27.4 & 15.6 & 11.1 & 10.9 & 15.1 & 20.0 & 17.3 & 18.6 & 33.9 & 15.3 & 27.4 & 15.0 & 19.6 & 31.1 & 31.5 & 31.5 & 47.3 \\
\hline NS2 & 15.6 & 15.7 & 17.0 & 16.1 & 15.8 & 15.8 & 16.8 & 17.9 & 17.8 & 17.8 & 17.5 & 18.8 & 17.5 & 15.3 & 14.3 & 32.2 & 29.9 & 29.9 & 41.9 \\
\hline NS3 & 37.2 & 38.9 & 37.5 & 39.2 & 35.9 & 37.6 & 36.7 & 35.6 & 35.6 & 38.8 & 38.1 & 41.2 & 37.6 & 37.3 & 40 & 46.6 & 48.1 & 48.3 & 53.0 \\
\hline NS4A & 17.6 & 25.5 & 21.6 & 26.9 & 23.5 & 25.5 & 19.6 & 17.3 & 21.2 & 15.4 & 21.2 & 27.5 & 25.0 & 23.5 & 29. & 42.3 & 28.8 & 28.8 & 46.2 \\
\hline NS4B & 21.6 & 21.8 & 22.0 & 21.8 & 20.2 & 16.8 & 17.5 & 22.1 & 22.4 & 24.1 & 23.5 & 19.4 & 24.8 & 23.2 & 19.3 & 35.0 & 32.4 & 32.4 & 41.6 \\
\hline NS5A & 12.0 & 11.9 & 10.4 & 11.7 & 15.7 & 11.6 & 10.8 & 12.3 & 12.3 & 13.6 & 11.4 & 9.2 & 12.1 & 15.5 & 9.8 & 19.7 & 12.1 & 11.7 & 27.2 \\
\hline NS5B & 31.6 & 31.6 & 31.1 & 34.0 & 31.4 & 30.9 & 32.1 & 32.1 & 33.0 & 34.4 & 34.2 & 34.6 & 35.1 & 32.0 & 32.2 & 56.3 & 43.1 & 43.3 & 54.8 \\
\hline
\end{tabular}

A to N indicate Hepacivirus A to Hepacivirus N, and RHV, JgV, HCL1, GD61, and BeHV indicate RHV-GS2015, Jogalong virus, DuHV-HCL1, DuHV-GD61, and Bald eagle hepacivirus, respectively. GenBank accession numbers for the sequences are as follows: Hepacivirus A, NC038425; Hepacivirus B, NC001655; Hepacivirus C, NC038882; Hepacivirus D, NC031950; Hepacivirus E, KC815310; Hepacivirus F, NC038427; Hepacivirus G, NC025672; Hepacivirus H, NC025673; Hepacivirus I, NC038428; Hepacivirus J, NC038429; Hepacivirus K, NC038430; Hepacivirus L, NC031916; Hepacivirus M, NC038431; Hepacivirus N, NC038432; RHV-GS2015, NC040815; Jogalong virus, MN133813; Bald eagle hepacivirus, MN062427; DuHV-HCL1, MK737640; DuHV-GD61, MT135177.

\subsection{Genomic Characterization of Hepacivirus $Q$}

The genome of Hepacivirus $Q$ consists of 9893 nucleotides and contains one large ORF encoding a polyprotein of 2999 aa. This polyprotein comprises considerably fewer amino acid residues than that of BeHV (3430 aa; MN062427) and other hepaciviruses identified 
in ducks (3607 aa; MK737639-MK737641 and MT135177). The putative cleavage sites specific for polyprotein processing were identified, and some of them were shown to be well conserved among the hepacivirus sequences analyzed here (Table 2). The predicted Hepacivirus $Q$ polyprotein contained ten proteins typical for hepaciviruses in the order of Core-E1-E2-p7-NS2-NS3-NS4A-NS4B-NS5A-NS5B (Figure 3A). The large ORF is flanked by $5^{\prime}$ and $3^{\prime}$ untranslated regions (UTRs) consisting of $585 \mathrm{nt}$ and $308 \mathrm{nt}$, respectively. Similar to $\mathrm{HCV}$ and other hepaciviruses, two and six $N$-glycosylation sites were also predicted in the putative E1 and E2 proteins of Hepacivirus $Q$, respectively (Figure 3A). Similar to other members of the genus Hepacivirus, a model Kozak sequence (AAGAUGG) at the proposed translation initiation site shows clear evidence of intrinsically disordered regions spanning the capsid protein and the $5^{\prime}$ half of NS5A [6]. Moreover, canonical microRNA 122 (miRNA-122) binding sites (CACUCC) were also absent in the $5^{\prime}$ NCR sequence of Hepacivirus $Q$, like other hepaciviruses identified in ducks [14].

Table 2. Comparison of predicted cleavage sites of hepacivirus polyprotein.

\begin{tabular}{|c|c|c|c|c|c|c|c|c|c|}
\hline \multirow[b]{2}{*}{ Virus } & \multicolumn{9}{|c|}{ Cleavage Site at: } \\
\hline & C/E1 & E1/E2 & $\mathrm{E} 2 / \mathrm{p} 7$ & P7/NS2 & NS2/NS3 & NS3/NS4A & NS4A/NS4B & NS4B/NS5A & NS5A/NS5B \\
\hline Hepacivirus A & GEA/SVV & VSC/DNY & AEA/YLS & AWA/FDN & RLL/SPI & TQT/NAW & EEC/FDH & QNC/DFT & ESC/SLS \\
\hline Hepacivirus B & CSG/ARV & IEA/TSG & MAA/GLP & ASA/FDT & AIT/APF & VNT/SGT & $\mathrm{EEC} / \mathrm{ASF}$ & DDC/GLI & FSC/SMS \\
\hline Hepacivirus C & ASA/YQV & VDA/ETH & AEA/ALE & AYA/LDT & RLL/API & VVT/STW & $\mathrm{EEC} / \mathrm{SQH}$ & TPC/SGS & VCC/SMS \\
\hline Hepacivirus D & GAS/CVV & VTS/TSL & AAA/AAM & AVG/FDD & MLN/PFS & NDC/SLV & EEC/SFG & AQC/DGG & AKC/ASW \\
\hline Hepacivirus E & ATA/VSN & AAA/AAP & AYA/FTP & AYA/ISL & KYT/IPF & FFA/SGY & EEC/YNW & DLC/TPP & HSC/SMS \\
\hline Hepacivirus F & GGA/VTN & VKA/LAL & AFA/FTP & SAY/SLN & ERT/APF & YFA/STT & EEC/YQW & EDC/SCR & HEC/SSW \\
\hline Hepacivirus G & ASA/GIF & VAA/PVS & VGA/LEV & EAY/EGG & $\mathrm{RFT} / \mathrm{APF}$ & YFA/ETV & EEC/STQ & DVC/TSP & TDC/SWS \\
\hline Hepacivirus H & AEA/NLL & SAV/AVP & SEA/VPT & RAE/QFD & QLT/KPF & YYC/GLV & $\mathrm{EEC} / \mathrm{ANE}$ & EIC/DGS & SSC/SKS \\
\hline Hepacivirus I & VEP/KPL & SVA/APV & YAQ/PPL & VEA/FSS & QLS/SPV & ELA/STW & EEC/ALD & EPC/TDS & ETC/TYS \\
\hline Hepacivirus J & AVS/HWC & AEG/LPF & ANA/LVL & AQG/GCL & RLT/APF & EEM/TDG & EEC/GFD & AEC/AGG & TSC/NYS \\
\hline Hepacivirus K & GEA/SYA & AQA/NPI & ADA/ALT & AVG/GPY & RHC/SPI & DDT/STG & EEC/LSY & $\mathrm{SEC} / \mathrm{AFF}$ & DEC/SAS \\
\hline Hepacivirus L & AES/VPA & AĀA/MPV & AWG/WPA & AQA/ASL & ERN/APM & YSA/GGL & EEC/MQT & $\mathrm{AEC} / \mathrm{DGM}$ & SKM/SRS \\
\hline Hepacivirus M & VDA/SFA & SQA/AEH & AEG/AME & VGG/GPV & RHC/SPI & TPT/SAW & EEC/ADY & RNC/SCS & SPC/SAS \\
\hline Hepacivirus N & VSG/YRQ & VEA/TTT & ATA/ALL & VTA/LDF & APC/SPI & LDV/WGA & $\mathrm{EEC} / \mathrm{WGF}$ & VPC/GFN & KEC/SYS \\
\hline $\mathrm{JgV}$ & AVA/FSD & AQA/GTH & IEG/AVN & VAG/FWF & KLA/API & SAG/LTV & EEC/AST & TNC/TSP & VCC/GES \\
\hline DuHV-HCL1 & ASA/DHI & GMA/DRS & AEG/MLS & VLG/ASV & QYT/API & NCS/AAY & $\mathrm{EEC} / \mathrm{SAE}$ & YEC/NSE & ESC/SFS \\
\hline DuHV-GD61 & ASA/DHI & GMA/DRS & AEG/MLS & VLG/ASV & QYT/API & NCS/AAY & EEC/SAE & YEC/NSE & ESC/SFS \\
\hline BeHV & ADS/SHD & VEG/GLQ & VTA/AVN & VSG/TEV & NWS/APL & VSC/SLY & EEC/AGN & YDC/ANS & HSC/SAS \\
\hline Hepacivirus Q & EPA/THL & VEG/GLV & VGA/AIN & VAG/SEI & RWS/APF & VNC/SML & EEC/SSS & QLC/SSN & CSC/SMS \\
\hline
\end{tabular}

Cleavage is indicated by a slash (/). The GenBank accession numbers for the corresponding sequences are same as shown in Table 1. 
A

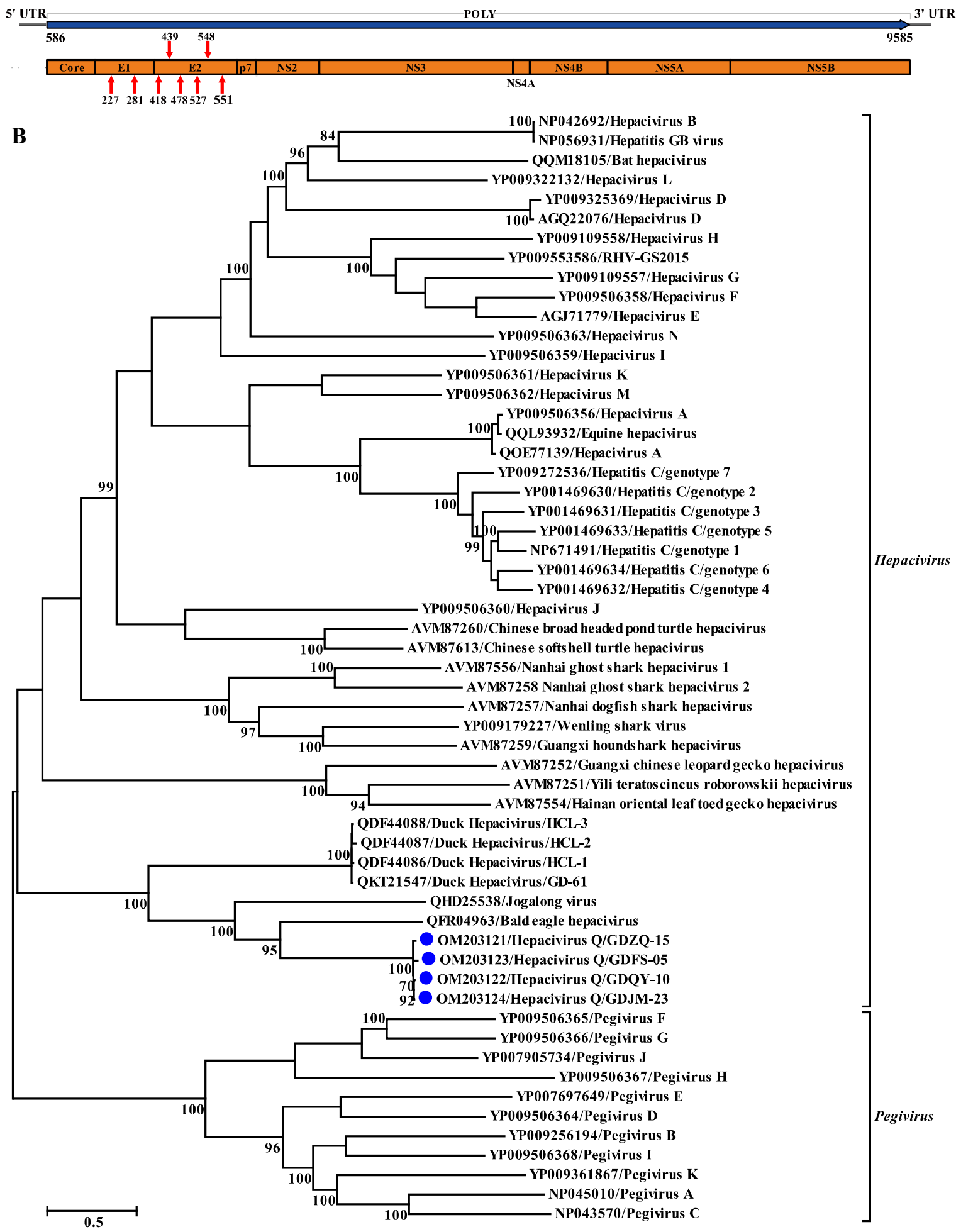

Figure 3. Genomic characterization and phylogenetic analysis based on the complete polyprotein of Hepacivirus $Q$ and other reference hepaciviruses. (A) Genome organization of Hepacivirus $Q$ identified in this study. Red arrows indicate N-linked glycosylation sites. (B) Phylogenetic analysis based on the amino acid sequence of the complete polyprotein of Hepacivirus $Q$. The trees were constructed based on the maximum likelihood method implemented in PhyML v3.0 and mid-point rooted for clarity; the scale bar represents the number of nucleotide substitutions per site. Bootstrap values were calculated with 100 replicates of the alignment, and only bootstrap values $>70 \%$ are shown at relevant nodes. 


\subsection{Prevalence and Genetic Diversity of Hepacivirus $Q$ in Guangdong}

To gain an insight into the prevalence of Hepacivirus $Q$ in duck populations, 270 individual duck liver samples collected from four duck farms located in Guangdong province, including those individual samples subjected to high-throughput RNA sequencing, were screened by nested RT-PCR using primers HepQ_NS5B_fwd1, HepQ_NS5B_fwd2, and HepQ_NS5B_rev (listed in Table S1). Using these primer pairs, a total of 43 positive individual samples collected from distinct duck farms were detected (Figure 1). The overall prevalence of Hepacivirus $Q$ in duck populations in Guangdong province was $15.9 \%$, while the prevalence in the four distinct duck farms ranged from $14.7 \%$ to $18.0 \%$. Four complete genome sequences of Hepacivirus $Q$ variants were obtained by overlapping nested RT-PCR and $5^{\prime} / 3^{\prime}$ RACE (primers listed in Table S1) from positive samples and have been submitted to the GenBank under accession numbers OM203121 to OM203124. The Hepacivirus $Q$ sequences identified herein showed low genome sequence diversity at the nucleotide level, ranging from 0.7 to $4.8 \%$. The pairwise amino acid distances indicated low divergence among the four Hepacivirus $Q$ sequences from this study but high divergence from other representative hepaciviruses (Table S3).

\subsection{Phylogenetic and Recombination Analyses of Hepacivirus $Q$}

Phylogenetic trees reconstructed based on the amino acid sequence of the complete polyprotein, NS3, and NS5B proteins support the grouping of the newly identified virus within the genus Hepacivirus. All the phylogenetic trees showed a similar topology such that the four Hepacivirus $Q$ strains formed a separate cluster distantly related to other hepaciviruses (Figures 3B and 4). Moreover, the Hepacivirus $Q$ strains branch very deeply next to DuHVs, BeHV, and the Jagolong virus $(\mathrm{JgV})$ and form the potential avian clade. Additionally, Hepacivirus $Q$ strains exhibited great genetic divergence from other duck hepaciviruses (HCL-1, -2, and -3, and GD-61), although they were also identified in ducks in China, suggesting the high genetic diversity of hepacivirus. Notably, Hepacivirus $Q$ strains formed a sister lineage with BeHV in the phylogenetic tree estimated based on the complete polyprotein. Still, they showed a closer relationship with BeHV and $\mathrm{JgV}$ in the NS3 and NS5B trees, respectively (Figures 3 and 4). The phylogenetic incongruence suggests that recombination events may have occurred in the evolutionary history of Hepacivirus $Q$, although no statistically supported recombination event was detected within Hepacivirus $Q$ strains or other hepaciviruses after systematic analyses. 


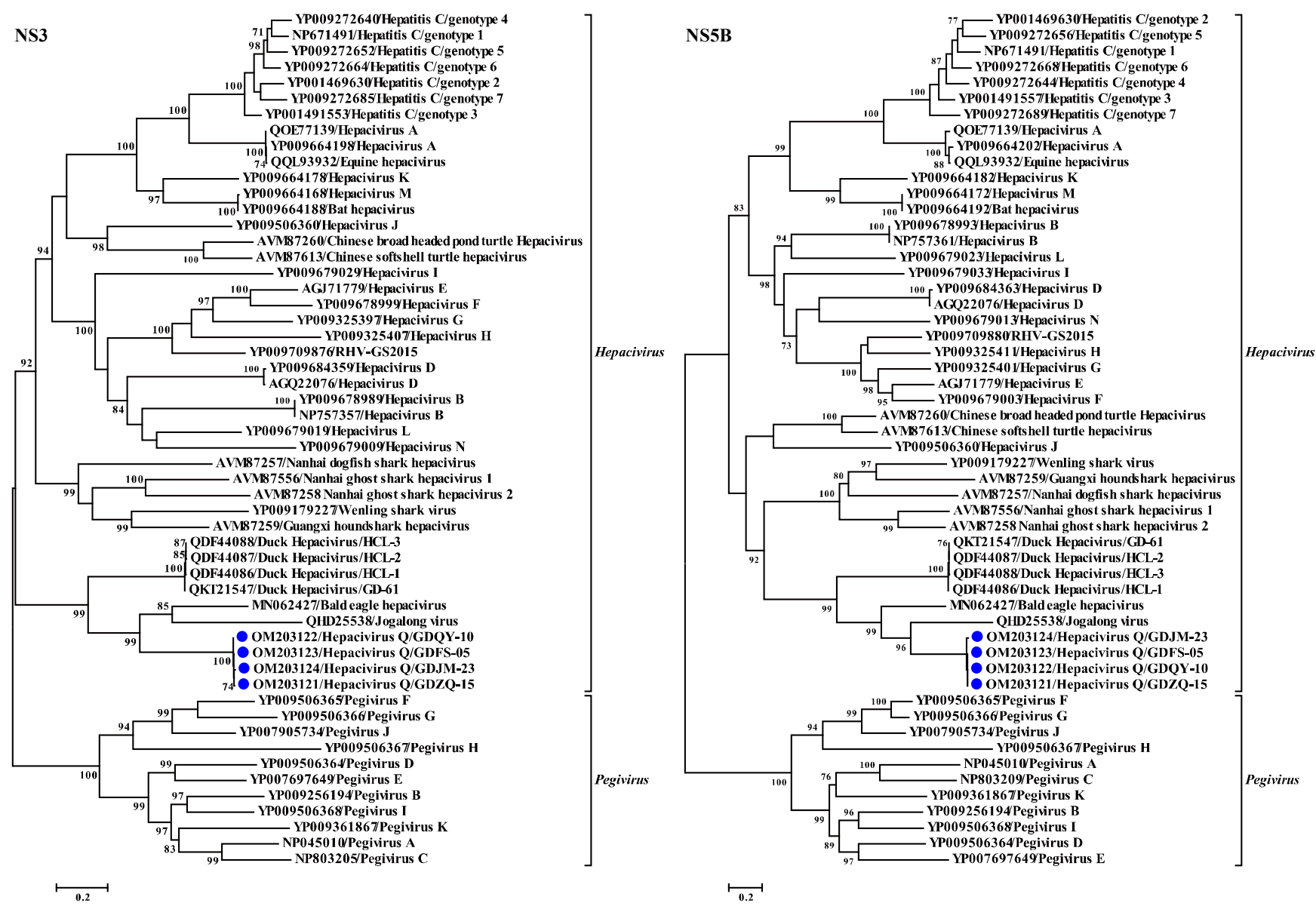

Figure 4. Phylogenetic analysis based on amino acid sequence of NS3 and NS5B proteins of hepaciviruses. The trees were constructed based on the maximum likelihood method implemented in PhyML v3.0 and mid-point rooted for clarity; the scale bar represents the number of nucleotide substitutions per site. Bootstrap values were calculated with 100 replicates of the alignment, and only bootstrap values $>70 \%$ are shown at relevant nodes.

\section{Discussion}

HCV has been considered as the only member of the genus Hepacivirus since its discovery in 1989. The first identification of hepaciviruses in dogs opened a window for a broad host spectrum of the genus Hepacivirus [36], and various hepaciviruses have been discovered from a wide range of host species [5-14,16-18], suggesting the ubiquitous presence of this highly variable virus. Here, a thus far unknown hepacivirus was identified in ducks and was named Hepacivirus Q. Estimation of evolutionary distances and phylogenetic analyses showing that the sequences of the four Hepacivirus $Q$ strains identified in different duck populations are closely related to each other but display large genetic distances from other previously described hepaciviruses suggest the extensive phylogenetic and host diversity of the genus Hepacivirus.

Hepacivirus $Q$ is a highly divergent hepacivirus and shares only $46.6 \%$ aa identity with its closest relative, Bald eagle hepacivirus, across the complete polyprotein. Noticeably, all the mature proteins of Hepacivirus $Q$ display a closer relationship with BeHV, except the most conserved protein, NS5B, which exhibits a higher sequence identity with $\mathrm{JgV}$ than BeHV (Table 1). Moreover, the incongruence displayed in the phylogenetic trees reconstructed based on the NS3 and NS5B proteins (Figure 4) also indicates that recombination events may have occurred in the evolutionary history of Hepacivirus $Q$. However, no statistically supported recombination event was detected within Hepacivirus $Q$ strains or other hepaciviruses after systematic analyses, although genetic recombination is an 
important evolutionary mechanism for HCV [37] and recombination events have been determined in other hepaciviruses [38,39], even in duck hepaciviruses [23]. Nevertheless, the large genetic distance from other known hepacivurses and the limitation of the data set hamper definite assertions on the presence or absence of recombination in Hepacivirus $Q$.

The vast majority of hepaciviruses identified to date are highly species-specific and are thought to be experiencing long-term coevolution and host adaptation [40]. In the present study, the phylogenetic placement of Hepacivirus Q with DuHVs, JgV, and BeHV indicates that they form a potential avian clade of hepacivruses, although they exhibit large genetic distances. In addition, the distant phylogenetic relationship between this potential avian clade and all other hepaciviruses indicates that hepaciviruses infecting avian hosts could be expected to diverge from mammalian, reptilian, or fish hepaciviruses, and also suggests a notably different evolutionary history for hepaciviruses in different host species.

As a human pathogen distributed worldwide, $\mathrm{HCV}$ poses a great threat to human health, causing liver failure, hepatitis, and hepatocellular carcinoma. Considerably diversified hepaciviruses have been identified in various animal species. However, animal hepaciviruses have been linked to clinical diseases in only a few cases [10,41]. Although duck hepacivirus was recently discovered in a study conducted to investigate the etiology of severely diseased ducks, the pathogenicity of duck hepacivirus is unclear as the virus was also highly prevalent in healthy ducks [14]. Similarly, Hepacivirus $Q$ was also identified in liver samples randomly collected from duck populations and it cannot be associated with any clinical disease. The high prevalence of Hepacivirus $Q$ in duck populations suggests that the virus may be non-pathogenic in ducks, and persistent infection may be established in the infected ducks. Future studies should investigate the epidemiology of Hepacivirus $Q$ in broader areas and populations and the pathogenicity of this novel virus in ducks.

In conclusion, a thus far unknown hepacivirus was identified in duck samples collected from Guangdong province of China through unbiased RNA sequencing, which expands knowledge concerning the genetic diversity and evolution of hepacivirues and indicates that genetically divergent hepaciviruses are circulating in duck populations in China.

Supplementary Materials: The following are available online at https: / / www.mdpi.com/article / 10.3390/v14020371/s1, Table S1: Primers used in this study, Table S2: Genetic divergence of Hepacivirus $Q$ compared to other hepaciviruses indicated by the amino acid $p$-distances in the conserved region of NS3 (positions 1123-1566) and NS5B (amino acid positions 2536-2959) as numbered in the reference sequence of Hepacivirus C (NC038882), Table S3: Pairwise amino acid distances among Hepacivirus $Q$ identified in this study and other hepaciviruses.

Author Contributions: Conceptualization, X.-L.Z. and J.-W.S.; methodology, X.-L.Z. and J.-W.S.; software, X.-L.Z. and J.-W.S.; formal analysis, X.-Y.Y. and J.-W.S.; resources, X.-Y.Y. and Y.-Q.Z.; investigation, X.-Y.Y., Z.-H.L., H.L., and J.S.; writing-original draft preparation, X.-L.Z.; writingreview and editing, J.-W.S.; visualization, J.-W.S.; supervision: X.-L.Z. and J.-W.S. All authors have read and agreed to the published version of the manuscript.

Funding: This study was supported by the Guangdong Basic and Applied Basic Research Foundation (2019A1515110157 and 2021A1515110450), Education Bureau of Guangdong province (2020ZDZX1004).

Institutional Review Board Statement: Ethical review and approval were waived for this study, all samples being received from the veterinary hospital of Foshan University and the samples being taken by authorized veterinarians in the frame of a farm visit for diagnostic purposes.

Data Availability Statement: The sequences generated in this study have been submitted to GenBank under accession numbers OM203121 to OM203124.

Conflicts of Interest: The authors declare no conflict of interest.

\section{References}

1. Simmonds, P.; Becher, P.; Bukh, J.; Gould, E.A.; Meyers, G.; Monath, T.; Muerhoff, S.; Pletnev, A.; Rico-Hesse, R.; Smith, D.B.; et al. ICTV virus taxonomy profile. J. Gen. Virol. 2017, 98, 2. [CrossRef] [PubMed]

2. Moradpour, D.; Penin, F.; Rice, C.M. Replication of hepatitis C virus. Nat. Rev. Microbiol. 2007, 5, 453-463. [CrossRef] [PubMed] 
3. Penin, F.; Dubuisson, J.; Rey, F.A.; Moradpour, D.; Pawlotsky, J.M. Structural biology of hepatitis C virus. Hepatology 2004, 39, 5-19. [CrossRef] [PubMed]

4. $\quad$ Boonstra, A.; van der Laan, L.J.; Vanwolleghem, T.; Janssen, H.L. Experimental models for hepatitis C viral infection. Hepatology 2009, 50, 1646-1655. [CrossRef] [PubMed]

5. Lyons, S.; Kapoor, A.; Sharp, C.; Schneider, B.S.; Wolfe, N.D.; Culshaw, G.; Corcoran, B.; McGorum, B.C.; Simmonds, P. Nonprimate hepaciviruses in domestic horses, United kingdom. Emerg. Infect. Dis. 2012, 18, 1976-1982. [CrossRef]

6. $\quad$ Lauck, M.; Sibley, S.D.; Lara, J.; Purdy, M.A.; Khudyakov, Y.; Hyeroba, D.; Tumukunde, A.; Weny, G.; Switzer, W.M.; Chapman, C.A.; et al. A novel hepacivirus with an unusually long and intrinsically disordered NS5A protein in a wild Old World primate. $J$. Virol. 2013, 87, 8971-8981. [CrossRef]

7. Quan, P.L.; Firth, C.; Conte, J.M.; Williams, S.H.; Zambrana-Torrelio, C.M.; Anthony, S.J.; Ellison, J.; Gilbert, A.T.; Kuzmin, I.V.; Niezgoda, M.; et al. Bats are a major natural reservoir for hepaciviruses and pegiviruses. Proc. Natl. Acad. Sci. USA 2013, 110, 8194-8199. [CrossRef]

8. Wu, Z.; Lu, L.; Du, J.; Yang, L.; Ren, X.; Liu, B.; Jiang, J.; Yang, J.; Dong, J.; Sun, L.; et al. Comparative analysis of rodent and small mammal viromes to better understand the wildlife origin of emerging infectious diseases. Microbiome 2018, 6, 178. [CrossRef]

9. Drexler, J.F.; Corman, V.M.; Müller, M.A.; Lukashev, A.N.; Gmyl, A.; Coutard, B.; Adam, A.; Ritz, D.; Leijten, L.M.; Van Riel, D.; et al. Evidence for novel hepaciviruses in rodents. PLoS Pathog. 2013, 9, e1003438. [CrossRef]

10. Baechlein, C.; Fischer, N.; Grundhoff, A.; Alawi, M.; Indenbirken, D.; Postel, A.; Baron, A.L.; Offinger, J.; Becker, K.; Beineke, A.; et al. Identification of a novel hepacivirus in domestic cattle from Germany. J. Virol. 2015, 89, 7007-7015. [CrossRef]

11. Corman, V.M.; Grundhoff, A.; Baechlein, C.; Fischer, N.; Gmyl, A.; Wollny, R.; Dei, D.; Ritz, D.; Binger, T.; Adankwah, E.; et al Highly divergent hepaciviruses from African cattle. J. Virol. 2015, 89, 5876-5882. [CrossRef] [PubMed]

12. Walter, S.; Rasche, A.; Moreira-Soto, A.; Pfaender, S.; Bletsa, M.; Corman, V.M.; Aguilar-Setien, A.; García-Lacy, F.; Hans, A.; Todt, D.; et al. Differential infection patterns and recent evolutionary origins of equine hepaciviruses in donkeys. J. Virol. 2017, 91, e01711-e01716. [CrossRef] [PubMed]

13. Shi, M.; Lin, X.D.; Vasilakis, N.; Tian, J.H.; Li, C.X.; Chen, L.J.; Eastwood, G.; Diao, X.-N.; Chen, M.-H.; Chen, X.; et al. Divergent viruses discovered in arthropods and vertebrates revise the evolutionary history of the flaviviridae and related viruses. J. Virol. 2016, 90, 659-669. [CrossRef] [PubMed]

14. Chu, L.; Jin, M.; Feng, C.; Wang, X.; Zhang, D. A highly divergent hepacivirus-like flavivirus in domestic ducks. J. Gen. Virol. 2019, 100, 1234-1240. [CrossRef] [PubMed]

15. Goldberg, T.L.; Sibley, S.D.; Pinkerton, M.E.; Dunn, C.D.; Long, L.J.; White, L.C.; Strom, S.M. Multidecade mortality and a homolog of hepatitis C virus in Bald eagles (Haliaeetus leucocephalus), the national bird of the USA. Sci. Rep. 2019, 9, 14953. [CrossRef]

16. Shi, M.; Lin, X.D.; Chen, X.; Tian, J.H.; Chen, L.J.; Li, K.; Wang, W.; Eden, J.S.; Shen, J.J.; Liu, L.; et al. The evolutionary history of vertebrate RNA viruses. Nature 2018, 556, 197-202. [CrossRef]

17. Porter, A.F.; Pettersson, J.H.; Chang, W.S.; Harvey, E.; Rose, K.; Shi, M.; Eden, J.S.; Buchmann, J.; Moritz, C.; Holmes, E.C. Novel hepaci- and pegi-like viruses in native Australian wildlife and non-human primates. Virus Evol. 2020, 6, veaa064. [CrossRef]

18. Chang, W.S.; Eden, J.S.; Hartley, W.J.; Shi, M.; Rose, K.; Holmes, E.C. Metagenomic discovery and co-infection of diverse wobbly possum disease viruses and a novel hepacivirus in Australian brushtail possums. One Health Outlook 2019, 1, 5. [CrossRef]

19. Williams, S.H.; Levy, A.; Yates, R.A.; Somaweera, N.; Neville, P.J.; Nicholson, J.; Lindsay, M.D.A.; Mackenzie, J.S.; Jain, K.; Imrie, A.; et al. Discovery of Jogalong virus, a novel hepacivirus identified in a Culex annulirostris (Skuse) mosquito from the Kimberley region of Western Australia. PLoS ONE 2020, 15, e227114. [CrossRef]

20. Harvey, E.; Rose, K.; Eden, J.S.; Lo, N.; Abeyasuriya, T.; Shi, M.; Doggett, S.L.; Holmes, E.C. Extensive diversity of RNA viruses in Australian Ticks. J. Virol. 2019, 93, e01358-18. [CrossRef]

21. Shao, J.W.; Guo, L.Y.; Yuan, Y.X.; Ma, J.; Chen, J.M.; Liu, Q. A novel subtype of bovine hepacivirus identified in ticks reveals the genetic diversity and evolution of bovine hepacivirus. Viruses 2021, 13, 2206. [CrossRef]

22. Li, L.L.; Liu, M.M.; Shen, S.; Zhang, Y.J.; Xu, Y.L.; Deng, H.Y.; Deng, F.; Duan, Z.J. Detection and characterization of a novel hepacivirus in long-tailed ground squirrels (Spermophilus undulatus) in China. Arch. Virol. 2019, 164, 2401-2410. [CrossRef] [PubMed]

23. Lu, G.; Zhao, J.; Ou, J.; Li, S. Novel HCV-Like virus detected in avian livers in Southern China and its implications for natural recombination events. Virol. Sin. 2021, 36, 149-151. [CrossRef] [PubMed]

24. Zhang, X.L.; Li, W.F.; Yuan, S.; Guo, J.Y.; Li, Z.L.; Chi, S.H.; Huang, W.J.; Li, X.W.; Huang, S.J.; Shao, J.W. Meta-transcriptomic analysis reveals a new subtype of genotype 3 avian hepatitis E virus in chicken flocks with high mortality in Guangdong, China. BMC Vet. Res. 2019, 15, 131. [CrossRef] [PubMed]

25. Chen, S.; Zhou, Y.; Chen, Y.; Gu, J. Fastp: An ultra-fast all-in-one FASTQ preprocessor. Bioinformatics 2018, 34, i884-i890. [CrossRef]

26. Li, D.; Liu, C.M.; Luo, R.; Sadakane, K.; Lam, T.W. MEGAHIT: An ultra-fast single-node solution for large and complex metagenomics assembly via succinct de Bruijn graph. Bioinformatics 2015, 31, 1674-1676. [CrossRef]

27. Buchfink, B.; Xie, C.; Huson, D.H. Fast and sensitive protein alignment using DIAMOND. Nat. Methods 2015, 12, 59-60. [CrossRef]

28. Langmead, B.; Salzberg, S.L. Fast gapped-read alignment with Bowtie 2. Nat. Methods 2012, 9, 357-359. [CrossRef]

29. Thorvaldsdóttir, H.; Robinson, J.T.; Mesirov, J.P. Integrative Genomics Viewer (IGV): High-performance genomics data visualization and exploration. Brief. Bioinform. 2013, 14, 178-192. [CrossRef] 
30. Li, C.X.; Shi, M.; Tian, J.H.; Lin, X.D.; Kang, Y.J.; Chen, L.J.; Qin, X.C.; Xu, J.; Holmes, E.C.; Zhang, Y.Z. Unprecedented genomic diversity of RNA viruses in arthropods reveals the ancestry of negative-sense RNA viruses. Elife 2015, 4, e05378. [CrossRef]

31. Katoh, K.; Standley, D.M. MAFFT multiple sequence alignment software version 7: Improvements in performance and usability. Mol. Biol. Evol. 2013, 30, 772-780. [CrossRef] [PubMed]

32. Guindon, S.; Gascuel, O. A simple, fast, and accurate algorithm to estimate large phylogenies by maximum likelihood. Syst. Biol. 2003, 52, 696-704. [CrossRef] [PubMed]

33. Darriba, D.; Taboada, G.L.; Doallo, R.; Posada, D. ProtTest 3: Fast selection of best-fit models of protein evolution. Bioinformatics 2011, 27, 1164-1165. [CrossRef] [PubMed]

34. Martin, D.P.; Murrell, B.; Golden, M.; Khoosal, A.; Muhire, B. RDP4: Detection and analysis of recombination patterns in virus genomes. Virus Evol. 2015, 1, vev003. [CrossRef]

35. Lole, K.S.; Bollinger, R.C.; Paranjape, R.S.; Gadkari, D.; Kulkarni, S.S.; Novak, N.G.; Ingersoll, R.; Sheppard, H.W.; Ray, S.C. Full-length human immunodeficiency virus type 1 genomes from subtype C-infected seroconverters in India, with evidence of intersubtype recombination. J. Virol. 1999, 73, 152-160. [CrossRef]

36. Kapoor, A.; Simmonds, P.; Gerold, G.; Qaisar, N.; Jain, K.; Henriquez, J.A.; Firth, C.; Hirschberg, D.L.; Rice, C.M.; Shields, S.; et al. Characterization of a canine homolog of hepatitis C virus. Proc. Natl. Acad. Sci. USA 2011, 108, 11608-11613. [CrossRef]

37. Galli, A.; Bukh, J. Comparative analysis of the molecular mechanisms of recombination in hepatitis C virus. Trends Microbiol. 2014, 22, 354-364. [CrossRef]

38. Lu, G.; Ou, J.; Sun, Y.; Wu, L.; Xu, H.; Zhang, G.; Li, S. Natural recombination of equine hepacivirus subtype 1 within the NS5A and NS5B genes. Virology 2019, 533, 93-98. [CrossRef]

39. Bletsa, M.; Vrancken, B.; Gryseels, S.; Boonen, I.; Fikatas, A.; Li, Y.; Laudisoit, A.; Lequime, S.; Bryja, J.; Makundi, R.; et al. Molecular detection and genomic characterization of diverse hepaciviruses in African rodents. Virus Evol. 2021, 7, veab036. [CrossRef]

40. Hartlage, A.S.; Cullen, J.M.; Kapoor, A. The strange, expanding world of animal hepaciviruses. Annu. Rev. Virol. 2016, 3, 53-75. [CrossRef]

41. Reuter, G.; Maza, N.; Pankovics, P.; Boros, A. Non-primate hepacivirus infection with apparent hepatitis in a horse-Short communication. Acta Vet. Hung. 2014, 62, 422-427. [CrossRef] [PubMed] 